\title{
El componente pragmático: elemento indispensable para la lectura en contexto*
}

\author{
Adriana María Palacio Medina \\ Institución Educativa José Acevedo y Gómez, Medellín, Colombia \\ adrypalamedi@hotmail.com \\ Bibiana Andrea Palacio Medina \\ Institución Educativa La Milagrosa, Medellín, Colombia \\ bivalent11@hotmail.com \\ Leysy Perea Mercado \\ Institución Educativa Sol de Oriente, Medellín, Colombia \\ leysy_perea@gmail.com \\ María Claribeth Moreno Romaña \\ Institución Educativa Luis Carlos Galán Sarmiento, Medellín, Colombia \\ clamoro28@hotmail.com
}

\section{RESUMEN}

Una de las prioridades, al finalizar el primer ciclo de educación primaria en Colombia, es que los estudiantes puedan reconocer la función social que cumplen los diferentes tipos de texto que leen. Para ello, dentro de los Lineamientos curriculares de lenguaje se aborda como proceso indispensable el desarrollo del componente pragmático referido al uso del lenguaje en contexto, aprendizaje necesario no solo para la vida escolar sino también para la vida social del niño. El objetivo de esta investigación fue analizar cómo se enseña la lectura desde el componente pragmático en el grado $3 .^{\circ}$ de cuatro instituciones educativas públicas de Medellín. Se utilizó una modalidad de investigación cualitativa bajo el enfoque histórico-hermenéutico y el método estudio de caso múltiple donde participaron ocho docentes que fueron entrevistadas y observadas en su práctica de aula. Para fundamentar esta investigación nos apoyamos en varios referentes teóricos: el componente pragmático referido al uso del lenguaje de María Victoria Escandell (1996), la competencia comunicativa desde la mirada de Dell Hymes (1984), la lectura como actividad social comunicativa de Teresa Colomer (2001), la función social de la lectura de Judith Kalman (2003) y el conocimiento disciplinar y didáctico de Andrés Perafán (2014).

Entre los resultados más relevantes, encontramos que las concepciones de las docentes hacen que en las prácticas de aula prioricen más los aspectos gramaticales de la lengua, además, se le da preferencia a los componentes semánticos y sintácticos y se deja de lado el pragmático. Hallamos que es predominante el uso de la narración en las prácticas lectoras frente a otras tipologías textuales, impidiendo de esta manera explorar otras formas de ver el contexto, además, las docentes requieren de conocimiento disciplinar y orientaciones curriculares sobre el componente pragmático.

Se puede concluir que, debido a que el rol docente está sujeto a los guiones y rutinas, el desarrollo de las clases de lectura se vuelve esquemático y dificulta la posibilidad de ampliar las perspectivas didácticas; además, la apropiación que hace el docente de todas estas orientaciones ministeriales no genera interacción con el saber científico, es decir, la pragmática. Igualmente, en las clases se deben abrir espacios donde los estudiantes practiquen las tipologías argumentativas como lo hacen en los espacios no escolares, pues se resalta que el aula de clase hace parte de los diferentes espacios sociales donde interactúan los alumnos dentro de su cotidianidad.

Palabras clave: componente pragmático; competencia comunicativa; lectura; tipología textual; conocimiento disciplinar y didáctico; función social de la lectura.

Cómo citar: Palacio Medina, A. M., Palacio Medina, B. A., Perea Mercado, L. y Moreno Romaña, M. C. (2018). El componente pragmático: elemento indispensable para la lectura en contexto. Ciencias Sociales y Educación, 7(14), 127-147. DOI: https://doi.org/10.22395/csye.v7n14a7

Recibido: 10 de junio de 2018.

Aprobado: 20 de octubre de 2018. 


\section{The Pragmatic Component: an Essential Element for Contextual Reading}

\section{ABSTRACT}

One of the priorities in the final stage of the first school cycle for a primary school in Colombia is that the students might be able to recognize the social function performed by different kinds of the texts they read. For this, the pragmatic component about the use of language in context is included within the language curriculum guidelines, resulting in a learning process not only for scholar life of the child but for its social life as well. The main objective of this research was analyzing how reading is taught from the pragmatic component in the third grade of for schools in Medellin. A qualitative method from a historical and hermeneutical approach was used alongside the case study method where eight teachers were interviewed and observed in its classroom practices. To substantiate this research we supported it with different theoretical references: the pragmatic component referred to the use of language from María Victoria Escandell (1996), the communicative competence from the point of view of Dell Hymes (1984), the social function of reading from Judith Kalman (2003) and the didactical and disciplinary knowledge from Andrés Perafán (2014). Among the most relevant results, we found that the conception the teach ers had made them prioritize the grammatical aspects of language and that semantic and syntactic components are preferred over the pragmatic ones. We found that narrative is the predominant type of text among other types and thus preventing the attempt of other ways of looking at context; furthermore, teachers require of disciplinary knowledge and curricular orientation about the pragmatic component.

We can conclude that given that the teacher's role is subject to scripts and routines, the development of reading classes becomes more schematic and thus it hinders the possibility of widening the didactical perspectives; besides, the assumption made by the teacher of the ministerial orientation doesn't interplay with scientific knowledge, i.e. pragmatics. In this same fashion, classes must be open spaces where students can put into action the argumentative typologies in the same way they do it in non-scholar contexts, given that it highlights that the classroom is part of the different social contexts in which students interact with each other in their everyday life.

Keywords: pragmatic component, communicative competence; reading; text typology; didactical and disciplinary knowledge; social function of reading.

\section{O componente pragmático: elemento indispensável para a leitura em contexto}

\section{RESUMO}

Reconhecer a função social que os diferentes gêneros textuais cumprem é uma das prioridades ao finalizar o primeiro ciclo da educação primária na Colômbia. Para isso, dentro dos Lineamentos curriculares de linguagem, aborda-se como processo indispensável para desenvolver o componente pragmático referente ao uso da linguagem em contexto, aprendizagem necessária não somente para a vida escolar, mas também para a vida social da criança. O objetivo desta pesquisa foi analisar como a leitura é ensinada a partir do componente pragmático do $3^{\circ}$ ano de quatro instituições educativas públicas de Medellín (Colômbia). Foi utilizada uma modalidade de pesquisa qualitativa sob a abordagem histórico-hermenêutica e o método estudo de caso múltiplo, do qual participaram oito docentes entrevistadas e observadas em sua prática de sala de aula. A pesquisa esteve apoiada em vários referenciais teóricos: o componente pragmático referente ao uso da linguagem de María Victoria Escandell (1996), a competência comunicativa sob a visão de Dell Hymes (1984), a leitura como atividade social comunicativa de Teresa Colomer (2001), a função social da leitura de Judith Kalman (2003) e o conhecimento disciplinar e didático de Andrés Perafán (2014).

Entre os resultados mais relevantes, verificamos que as concepções das docentes fazem com que, nas práticas de sala de aula, sejam priorizados mais os aspectos gramaticais da língua; além disso, dão preferência aos componentes semânticos e sintáticos e deixam de lado o pragmático. Constatamos que é predominante o uso da narração nas práticas de leitura ante outros tipos textuais, o que impede explorar outras formas de ver o contexto; ainda, as docentes requerem conhecimento disciplinar e orientações curriculares sobre o componente pragmático. Pode-se concluir que, devido ao papel do docente estar sujeito aos programas e às rotinas, o desenvolvimento das aulas de leitura se torna esquemático e dificulta a possibilidade de ampliar as perspectivas didáticas; além disso, a apropriação que o docente faz de todas essas orientações não gera interação com o saber científico, isto é, a pragmática. Ainda, nas aulas, devem ser abertos espaços em que os estudantes pratiquem os tipos argumentativos como fazem em espaços não escolares, pois se ressalta que a sala de aula faz parte dos diferentes espaços sociais onde os alunos interagem dentro do seu cotidiano.

Palavras-chave: componente pragmático; competência comunicativa; leitura; tipo textual; conhecimento disciplinar e didático; função social da leitura. 


\section{Introducción}

La lectura es una práctica social que está presente en todos los espacios donde se desenvuelven las personas, siendo un factor esencial para el desarrollo del pensamiento, la creatividad, la reflexión y la participación. El componente pragmático de la lectura está relacionado con la intencionalidad de lo que se dice, por qué, a quién y para qué se dice; es decir, el contexto. Los Estándares básicos de competencias en Colombia establecen que, al finalizar el primer ciclo de primaria (grado . $^{\circ}$ ), los estudiantes deben "comprender textos que tengan diferentes formatos y finalidades" (Ministerio de Educación Nacional, 2006). En otras palabras, reconocer su función social. Sin embargo, en las prácticas de aula, a menudo se encuentran dificultades en el componente pragmático, pues a los estudiantes les cuesta identificar información implícita y reconocer la función en contexto de los diferentes tipos de texto que leen. Las pruebas estandarizadas aplicadas por el Icfes en los últimos tres años (2015, 2016 y 2017) demuestran que los estudiantes de las instituciones educativas José Acevedo y Gómez, La Milagrosa, Luis Carlos Galán Sarmiento y Sol de Oriente poseen desempeños insuficientes en este componente.

En el entorno escolar, a menudo encontramos varios factores que podrían ser una causa de estos resultados desfavorables en el componente pragmático, los cuales son: las docentes generan pocos espacios de comprensión de elementos implícitos y explícitos del acto comunicativo; los estudiantes tienen dificultad para reconocer y analizar información y elementos explícitos e implícitos sobre los propósitos del texto; manifiestan desinterés por las prácticas de lectura en el aula y no comprenden suficientemente lo que leen, lo cual les impide relacionar conocimientos, aprender con facilidad y aplicar dichos saberes en una situación comunicativa.

Las prácticas de lectura son, sin duda, una de las mayores preocupaciones en investigaciones educativas. En el proceso de rastreo se hallaron diversas investigaciones, no explícitamente del componente pragmático, pero sí de la competencia comunicativa en el que está incluido. En su mayoría, estas investigaciones analizan las prácticas de lectura y escritura en el aula, y la creación de estrategias metodológicas que permitan a los estudiantes integrar el contexto en los procesos de la lectura y la escritura, para mejorar su competencia comunicativa. Cabe resaltar que, en el área de lenguaje, el componente pragmático ha sido abordado insuficientemente en las investigaciones educativas, por tanto, se hace necesario profundizar su estudio, promoción y aplicación dentro del contexto escolar.

La investigación se realizó con ocho docentes del grado $3 .^{\circ}$ de las cuatro instituciones educativas participantes, las cuales se encuentran ubicadas en 
la ciudad de Medellín (Colombia). Para llevarla a cabo se planteó como objetivo general analizar las prácticas de enseñanza de la lectura desde el componente pragmático en el grado 3. de las instituciones educativas José Acevedo y Gómez, La Milagrosa, Luis Carlos Galán Sarmiento y Sol de Oriente. Para darle cumplimiento a los objetivos, nos apoyamos en un paradigma cualitativo, con enfoque de tipo historicohermenéutico, el cual nos permitió reflexionar sobre nuestra práctica pedagógica. En el contexto de la investigación se eligió el método estudio de caso múltiple para tener una mirada del contexto en el que se desarrolló la propuesta investigativa.

Las categorías que se abordaron en la investigación fueron la competencia comunicativa, el componente pragmático, la función social de la lectura y el conocimiento disciplinar y didáctico. La competencia comunicativa, porque su desarrollo posibilita que una persona se proyecte ante la sociedad, demuestre coherencia entre lo que piensa y lo que expresa, tenga la capacidad para mantener el tema de la conversación y se adapte al contexto, es decir, a la situación comunicativa de la que está participando. El componente pragmático de la lectura porque está relacionado con la intencionalidad de lo que se dice, por qué, a quién y para qué se dice; es decir, el contexto y todos los componentes materiales e inmateriales que intervienen en la comunicación. La función social de la lectura, ya que permite reflexionar que un proceso lector debe ir más allá y tocar un plano no sólo didáctico y pedagógico sino también social, pues los libros por sí solos no promueven la lectura, es su uso y apropiación lo que define esa situación. Se debe entender que en la lectura juega un papel fundamental no solo el texto, sino también el contexto, el mensaje y los lectores. El conocimiento disciplinar y didáctico porque muestra la importancia de reconocer a los docentes como parte activa en la producción del saber disciplinar en la escuela, pues son los agentes que, a través de su conocimiento, permiten en sus estudiantes el desarrollo de habilidades y competencias para la vida.

\section{Materiales y métodos}

La presente investigación es cualitativa, ofrece pautas para la interpretación, comprensión y conocimiento del medio en el que se encuentra inmersa la comunidad educativa. Además, le permite al docente orientar y renovar su práctica pedagógica por medio de una observación en la cual este no solo investiga su labor, sino que va elaborando procesos de transformación.

Este tipo de investigación permite una reflexión constante de la práctica pedagógica, al mismo tiempo que sirve de motivación para que los docentes nos formemos y nos actualicemos en las diferentes teorías de enseñanza y aprendizaje para que los estudiantes alcancen aprendizajes significativos y 
contextualizados. La investigación cualitativa se sustenta por medio de enfoques que le permiten darle identidad a la investigación, pero a la vez facilitan que el investigador se oriente, conozca, explore, transforme su realidad y pueda pensar de manera precisa en ciertos enfoques que conducen la participación investigativa al detalle, es por esto que para esta propuesta se ha pensado en un enfoque de tipo historicohermenéutico, el cual:

Busca reconocer la diversidad, comprender la realidad; construir sentido a partir de la comprensión histórica del mundo simbólico; de allí el carácter fundamental de la participación y el conocimiento del contexto como condición para hacer la investigación. No se puede comprender desde afuera, desde la neutralidad; no se puede comprender algo de lo que no se ha participado. Se busca comprender el quehacer, indagar situaciones, contextos y particularidades, simbologías, imaginarios, significaciones, percepciones, narrativas, cosmovisiones, sentidos, estéticas, motivaciones, interioridades, intenciones que se configuran en la vida cotidiana. La vivencia y el conocimiento del contexto, así como experiencias y relaciones, se consideran como una mediación esencial en el proceso de conocimiento, que se tiene en cuenta al diseñar el proyecto de investigación (Cifuentes, 2011, p. 30).

En el contexto de la investigación se eligió el método de estudio de casos múltiple, el cual nos permite tener una mirada del contexto en el que se desarrolla la propuesta investigativa y en la que el investigador puede estar en contacto con la población a estudiar. Además, es un método que permite involucrar a varias instituciones, teniendo como objeto de estudio las prácticas de enseñanza que comprenden el componente pragmático en la lectura. Los referentes contextuales de estas instituciones se presentan a continuación.

La Institución Educativa José Acevedo y Gómez, ubicada en el barrio Guayabal, de la Comuna 15 de Medellín, Antioquia. Es una institución de carácter oficial que se ha enfocado en la inclusión escolar. Por esta razón, a través del modelo pedagógico crítico social, busca que toda la comunidad educativa se concientice de la importancia del respeto por la diferencia y del verdadero sentido de la autonomía.

La Institución Educativa La Milagrosa, ubicada en el parque principal del barrio La Milagrosa, de la Comuna 9 de Medellín, Antioquia. Es de carácter oficial y presta sus servicios educativos en los niveles de preescolar, básica primaria, básica secundaria y media técnica. Esta institución tiene como compromiso abordar una metodología del conocer, ser y hacer, formando jóvenes competentes, estimulando el ser desde el humanismo y la tecnología. Las orientaciones están encaminadas a formar personas integrales, que puedan enfrentarse al mundo de manera autónoma y crítica. El modelo pedagógico de la institución se encuentra en proceso de construcción.

La Institución Educativa Luis Carlos Galán Sarmiento, ubicada en el barrio Enciso, Comuna 8 de Medellín, Antioquia. Es pública, de carácter mixto. El 
modelo pedagógico es el social, con el cual pretende contribuir a la transformación social de los estudiantes, formando parte activa en la construcción del conocimiento.

La Institución Educativa Sol de Oriente, localizada en la Comuna 8 de la ciudad de Medellín. Su modelo pedagógico es el holístico, ya que permite el desarrollo del ser, el saber, el conocer, el hacer y el aprender a hacer.

Estas instituciones educativas tienen en común que su población estudiantil pertenece a los estratos 0,1 y 2 ; las cuatro instituciones pretenden formar a los niños, niñas y jóvenes a través de un proyecto educativo que estimule el desarrollo integral basado en el respeto, la convivencia y tolerancia, el cual contribuya a la transformación social. También tienen en común que han presentado dificultades en los resultados de pruebas Saber, por lo que cuentan con el apoyo del programa Todos a aprender para el fortalecimiento de los procesos lectoescriturales y logicomatemáticos. Lo que diferencia a las cuatro instituciones son sus modelos pedagógicos.

\section{Población y muestra}

La investigación se realizó con ocho docentes del grado 3. de las instituciones educativas José Acevedo y Gómez, La Milagrosa, Luis Carlos Galán Sarmiento y Sol de Oriente, las cuales fueron seleccionadas de manera intencional, ya que laboraban en jornada contraria a la de las investigadoras, lo cual permitió que las directivas de las instituciones otorgaran con mayor facilidad los permisos para la realización de las observaciones y los talleres pedagógicos. Se destaca que, aunque la investigación se realizó con las docentes del grado $3 .^{\circ}$, los talleres pedagógicos fueron aplicados a todos los docentes del primer ciclo de básica primaria (cincuenta y dos docentes), para tener un mayor impacto en la población beneficiada. Las edades de las docentes oscilan entre los 42 y los 65 años, todas con más de dieciséis años de experiencia en el sector público, además, son licenciadas en diferentes disciplinas de la educación, la mayoría son especialistas y solo una es magíster.

\section{Consideraciones éticas}

Se tuvo como propósito fundamental seguir una ruta metodológica que permitiera recopilar información fidedigna, pertinente y transparente, a través de cuatro técnicas de recolección de información junto con sus respectivos instrumentos, previamente validados. Las técnicas utilizadas fueron: la revisión documental, la observación, la entrevista semiestructurada y los talleres pedagógicos. Los espacios para la aplicación de los mismos fueron autorizados por las directivas de las instituciones y concertados con las docentes participantes en la investigación en donde se firmó un consentimiento informado por cada una de ellas. 


\section{Fases metodológicas}

Inicialmente se hizo un rastreo de antecedentes, conceptos y teorías acerca del componente pragmático de la lectura, se identificó el problema, se eligió una metodología de investigación bajo la modalidad cualitativa, con un enfoque histórico-hermenéutico y el método es el estudio de caso múltiple. Las técnicas de recolección de información fueron: revisión documental (resultados pruebas Saber 2015, 2016 y 2017, los lineamientos y mallas curriculares, los Estándares básicos de competencias, los Derechos básicos de aprendizaje, la matriz de referencia lenguaje, el libro Lectura y composición en español del programa PTA, las guía de aplicación para las pruebas Saber 2015, 2016 y 2017), la observación (se observaron dos clases a ocho docentes de las cuatro instituciones educativas participantes), entrevista semiestructurada (participaron ocho docentes del grado $3 .^{\circ}$ de básica primaria) talleres pedagógicos (se diseñaron tres talleres a cincuenta y dos docentes del primer ciclo de básica primaria de las cuatro instituciones educativas participantes). La metodología de investigación se desarrolló en tres fases así:

Fase número 1. Revisión documental: se realizó un rastreo bibliográfico de documentos oficiales del Ministerio de Educación Nacional que permitieron reflexionar sobre el impacto que tienen las orientaciones ministeriales de lengua castellana y el resultado de las pruebas Saber del grado 3..$^{\circ}(2015,2016$ y 2017) en las prácticas de enseñanza de los docentes participantes en la investigación. El tiempo destinado para esta fase fue de tres semestres, ya que como lo sustenta la investigación cualitativa se debe realizar una retroalimentación de manera constante.

Fase número 2. Observación y entrevista semiestructurada: para identificar cuáles fueron las estrategias que utilizan los docentes del grado $3 .^{\circ}$ para la enseñanza de la lectura en el componente pragmático, se utilizaron dos instrumentos de recolección información (observación y entrevista semiestructurada).

- Observación. Se elaboró una guía de observación con trece aspectos, los cuales abordaron las cuatro categorías centrales de análisis (competencia comunicativa, componente pragmático, función social de la lectura, conocimiento disciplinar y didáctico). Para codificar la información recolectada se elaboró una ficha de contenido en donde se analizaron los ítems de la observación teniendo en cuenta las categorías mencionadas.

- Entrevista semiestructurada. Se elaboró una guía de entrevista con doce preguntas que tenían como objetivo identificar los conocimientos que tienen los docentes del grado sobre el componente pragmático y su aplicabilidad en el aula. Antes de aplicar la entrevista, se realizó una prueba piloto con docentes que no participaron en la investigación, con el fin de identificar 
posibles sesgos en su elaboración. Al llevar a cabo esta estrategia nos dimos cuenta de que debíamos hacer en primer lugar las observaciones y luego las entrevistas, ya que las docentes podían apropiarse de la temática y posiblemente sesgar la información necesaria para la investigación. Para agrupar los datos, tomamos las respuestas de las entrevistas y las codificamos teniendo en cuenta las categorías. La información recolectada fue organizada en fichas de contenido, las cuales sirvieron de apoyo para la triangulación de la información.

Fase número 3. Talleres pedagógicos: se diseñaron tres talleres pedagógicos que sirvieron de apoyo para orientar las prácticas de enseñanza de la lectura en el componente pragmático, no solo a las docentes de $3 .^{\circ}$ que estaban participando en la investigación, sino a todos los docentes del primer ciclo de básica primaria de las instituciones educativas involucradas (cincuenta y dos docentes). El tiempo de diseño, elaboración, aplicación y sistematización de los talleres fue de aproximadamente cincuenta horas por taller. Los talleres pedagógicos puestos en práctica constaron de cuatro momentos: actividades introductorias, desarrollo del tema, recapitulación y cierre.

Antes de aplicar los talleres se realizaron actividades de sensibilización, para que los docentes se fueran contextualizando con la temática a tratar. Con ocho días de anticipación se entregó a cada uno de los cincuenta y dos docentes participantes una tarjeta de invitación informando los objetivos de los talleres, el lugar, las horas y fechas en donde se realizaría el encuentro; también se instalaron en diferentes lugares de las instituciones letreros publicitarios con ejemplos y definiciones.

En los cierres de cada uno de los talleres se realizó una evaluación, la cual sirvió para identificar el alcance de los objetivos y las sugerencias que los docentes tenían para el siguiente encuentro. Estas fueron sistematizadas en fichas de registro, las cuales permitieron registrar y analizar la información.

\section{Resultados}

Caracterización pruebas Saber 2015, 2016, 2017

Con respecto a la prueba de lenguaje que se aplica en las pruebas Saber, el MEN indica que:

La prueba de lenguaje evalúa dos competencias: la comunicativa-lectora y la comunicativa-escritora. La competencia comunicativa-lectora, abarca la comprensión, el uso y la reflexión sobre las informaciones contenidas en diferentes tipos de textos, e implica una relación dinámica entre estos y el lector y considera tres componentes transversales: el sintáctico, el semántico y el pragmático (2006, pp. 16-17). 
Según la guía de aplicación de prueba de lenguaje del Icfes en los años 2015, 2016 y 2017, para evaluar el componente pragmático de la lectura se tienen en cuenta los siguientes aspectos: que el estudiante pueda "reconocer información explícita sobre los propósitos del texto, reconocer elementos implícitos sobre los propósitos del texto, analizar información explícita o implícita sobre los propósitos del texto" (MEN, 2016, pp. 19-20). En la prueba estandarizada para todas las instituciones educativas de Colombia, el porcentaje de preguntas aplicadas del componente pragmático de la lectura fue de un $14 \%$ de la prueba, en el año 2016 un 12 \% de la prueba y en el año 2017 un promedio del 11,3\%.

Según los resultados de estos últimos tres años, en las cuatro instituciones participantes, los aprendizajes a mejorar son todos en torno al componente pragmático (tabla 1).

Tabla 1. Aprendizajes por mejorar

\begin{tabular}{ccccc}
\hline Institución Educativa & 2015 & 2016 & 2017 & Aprendizajes por mejorar \\
\hline José Acevedo y Gómez & $78 \%$ & $52 \%$ & $44 \%$ & $\begin{array}{l}\text { No reconoce o evalúa la información implícita } \\
\text { y explícita en una situación de comunicación. }\end{array}$ \\
\hline
\end{tabular}

No reconoce elementos implícitos de un tex-

Sol de Oriente $\quad 77 \% \quad 77 \% \quad 43 \% \begin{aligned} & \text { to y no evalúa la información implícita y ex- } \\ & \text { plícita en una situación de comunicación, ni }\end{aligned}$ reconoce su estructura explícita.

No recupera información implícita de un texLuis Carlos Galán to, no reconoce la estructura implícita ni exSarmiento $\quad 70 \% \quad 61 \% \quad 60 \% \quad$ plícita de un texto y no evalúa la informacion implícita y explícita en una situación de comunicación.

$\begin{array}{lllll}6 & 66 \% & 79 \% & 69 \% & \begin{array}{l}\text { No reconoce la estructura implícita y no eva- } \\ \text { lúa la informacion implícita y explícita en una } \\ \text { situación de comunicación. }\end{array}\end{array}$

Fuente: resultados pruebas Saber 2015, 2016 y 2017 de las instituciones educativas participantes en la investigación.

Los lineamientos curriculares de la lengua castellana indican que:

En el primer ciclo de primaria se debe hacer mayor énfasis en el trabajo sobre la construcción y el uso de los sistemas de significación, más que a la explicación del funcionamiento del sistema. Es decir, el manejo de categorías lingüísticas que den cuenta de la lengua como objeto deben ser trabajados sobre los usos sociales y culturales del lenguaje, y no como una teorización en abstracto (MEN, 1998, p. 19).

Sin embargo, podemos evidenciar que el porcentaje de preguntas de tipo pragmático aplicadas en los últimos tres años es mínimo en relación con los 
componentes semántico y sintáctico que corresponde al resto de la ponderación y que año tras año ha disminuido, lo que también puede a su vez explicar por qué en el último año todas las instituciones mostraron mejoría. Lo que se debe hacer es continuar fortaleciendo este componente, en todos los procesos de enseñanza aprendizaje, ya que los aspectos sociales y contextuales, juegan un papel indispensable en la comunicación.

A continuación, se presentan los resultados de las observaciones y las entrevistas, los cuales se analizaron en fichas de contenido y fueron agrupados teniendo en cuenta cada una de las categorías de la investigación.

Tabla 2. Observación y entrevista semiestructurada

\begin{tabular}{|c|c|c|}
\hline Categoría & Resultados de la observación & $\begin{array}{l}\text { Resultados de la entrevista } \\
\text { semiestructurada }\end{array}$ \\
\hline & $\begin{array}{l}\text { El docente utiliza el diálogo para } \\
\text { contribuir a la comprensión del texto }\end{array}$ & $\begin{array}{l}\text { ¿Qué es para usted, la competencia } \\
\text { comunicativa? }\end{array}$ \\
\hline & $\begin{array}{l}\text { El } 94 \% \text { de las docentes generó si- } \\
\text { tuaciones dialógicas en las cuales } \\
\text { les permitían a sus estudiantes es- } \\
\text { tablecer interlocuciones y compren- } \\
\text { der información literal de los textos } \\
\text { empleados, su competencia comuni- }\end{array}$ & $\begin{array}{l}\text { El } 75 \% \text { de las docentes entrevistadas } \\
\text { definen la competencia comunicativa } \\
\text { como la capacidad que tiene una per- } \\
\text { sona para comunicarse asertivamen- } \\
\text { te, mientras que el } 25 \% \text {, expresa que } \\
\text { es el conjunto de las habilidades del }\end{array}$ \\
\hline $\begin{array}{l}\text { Competencia } \\
\text { comunicativa }\end{array}$ & $\begin{array}{l}\text { cativa permitía que los juicios brin- } \\
\text { dados en estos diálogos permitieran } \\
\text { un mayor acercamiento a la temáti- } \\
\text { ca trabajada en clase. El } 6 \% \text { de los } \\
\text { docentes no generaron situaciones } \\
\text { dialógicas con los estudiantes. Es } \\
\text { así como "las docentes emplearon } \\
\text { el diálogo para el desarrollo de sus } \\
\text { clases, lo que permitió una partici- } \\
\text { pación activa por parte de los estu- } \\
\text { diantes" (fragmento observación de } \\
\text { clase I. E. Sol de Oriente). }\end{array}$ & $\begin{array}{l}\text { lenguaje (leer, escribir, hablar y es- } \\
\text { cuchar). Se evidencia un acercamien- } \\
\text { to por parte de las docentes a lo que } \\
\text { nos expresa Hymes (1984) acerca de } \\
\text { lo que se define como competencia } \\
\text { comunicativa, pero un grupo de do- } \\
\text { centes necesitan pasar de un aspec- } \\
\text { to fundamental como son las habili- } \\
\text { dades comunicativas, a un concepto } \\
\text { amplio como lo es la competencia co- } \\
\text { municativa. }\end{array}$ \\
\hline
\end{tabular}

¿Cuáles son los componentes que evalúa el Icfes desde la competencia comunicativa?

El $75 \%$ de docentes entrevistadas no tienen claro cuáles son los componentes que evalúa el Icfes en la prueba Saber de lenguaje. Podemos observar que un alto porcentaje de docentes desconocen los componentes evaluados en las pruebas Saber por el Icfes. 
El $25 \%$ tienen conocimiento de los componentes que evalúa el Icfes desde la competencia comunicativa.

¿A cuál de los componentes de la competencia comunicativa le da más importancia en su planeación y por qué?

El $50 \%$ de las docentes entrevistadas consideran importante los tres componentes (semántico, sintáctico, pragmático). El otro $50 \%$ no tiene claridad sobre cuáles son estos componentes, lo que se evidencia en sus repuestas desligadas del tema central. Como se evidencia en las respuestas de las docentes, la mitad de las entrevistadas reconocen los componentes propios de la competencia comunicativa y tratan de incorporarlos dentro de sus planeaciones, el otro porcentaje de docentes, al desconocerlos no permiten que sus estudiantes desarrollen dichos componentes, ya que no se incorporan dentro de sus planeaciones.

El docente, al desarrollar las estrategias de lectura, lleva al estudiante a identificar información explícita sobre los propósitos del texto.

El $75 \%$ de las docentes observadas no implementó estrategias que llevaran a sus estudiantes a identificar información explícita de los textos abordados en la clase, "las docentes observadas realizan preguntas

Componente pragmático textuales sobre el contenido de los textos. No se evidencian preguntas sobre el propósito del texto" (fragmento observación de clase I. E. Luis Carlos Galán Sarmiento). El 25 \% restante utilizó preguntas que permitieron identificar el propósito del texto.

El docente al desarrollar las estrategias de lectura lleva al estudiante a identificar información implícita sobre los propósitos del texto.
¿Qué entiende por componente pragmático desde la competencia comunicativa?

El $62 \%$ de las docentes demostraron poco conocimiento teórico referente al componente pragmático desde la competencia comunicativa. Ejemplo de respuesta "E1S: Creo que es la parte de las preguntas que no están en un texto implícitas".

El 38 \% de los docentes reconocen algunos elementos del componente pragmático desde la competencia comunicativa. 


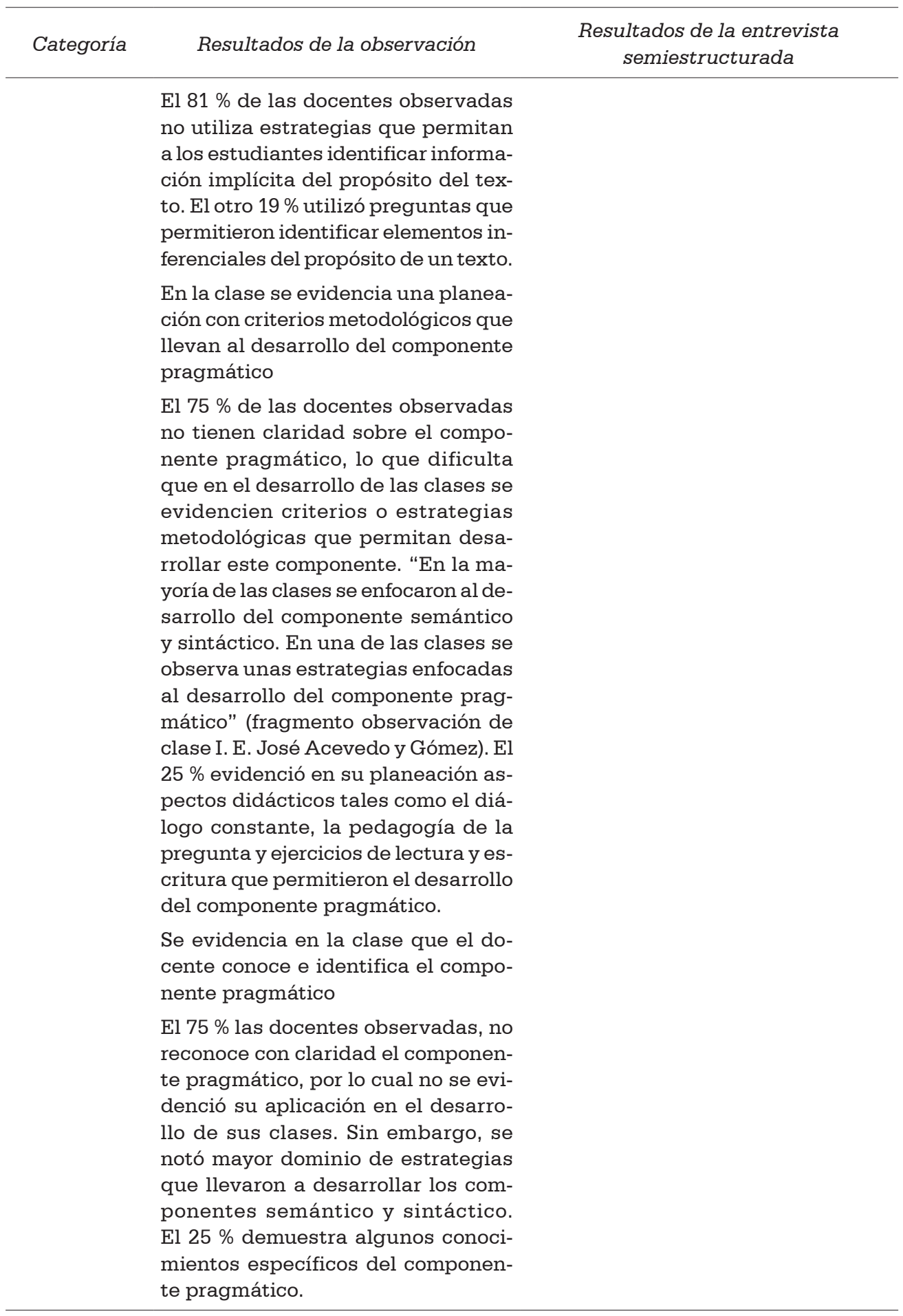




\section{Categoría Resultados de la observación}

Resultados de la entrevista semiestructurada

\begin{tabular}{|c|c|c|}
\hline $\begin{array}{l}\text { Función } \\
\text { social de la } \\
\text { lectura }\end{array}$ & $\begin{array}{l}\text { El docente establece relaciones entre } \\
\text { el contenido del texto y el contexto } \\
\text { En el } 70 \% \text { de las clases observadas, } \\
\text { las docentes establecieron relacio- } \\
\text { nes entre el contenido del texto y el } \\
\text { contexto. "En las clases observadas } \\
\text { las docentes trasladan algunas ex- } \\
\text { presiones del texto a la vida cotidia- } \\
\text { na de los estudiantes, hacen pregun- } \\
\text { tas sobre la relación de lo leído con el } \\
\text { contexto, resaltando algunas expre- } \\
\text { siones propias de la región antioque- } \\
\text { ña" (fragmento observación de cla- } \\
\text { se I. E. Luis Carlos Galán Sarmiento). } \\
\text { Sin embargo, en el } 30 \% \text { de las clases } \\
\text { no se evidenció este proceso. }\end{array}$ & $\begin{array}{l}\text { ¿Qué estrategia utiliza para integrar } \\
\text { la lectura que realiza en el aula con el } \\
\text { contexto de los estudiantes? } \\
\text { Las estrategias utilizadas por el } 87 \% \\
\text { de las docentes para integrar las lec- } \\
\text { turas al contexto de los estudiantes } \\
\text { son: intereses de los estudiantes, ex- } \\
\text { ploración de saberes previos, diálogo } \\
\text { sobre sus experiencias relacionadas } \\
\text { con el texto y proposición de solucio- } \\
\text { nes a problemas de su entorno. El } 13 \\
\% \text { no responde. }\end{array}$ \\
\hline $\begin{array}{l}\text { Conocimien- } \\
\text { to disciplinar } \\
\text { y didáctico }\end{array}$ & $\begin{array}{l}\text { Tipología textual utilizada en la cla- } \\
\text { se } \\
\text { El texto narrativo fue empleado en el } \\
62 \% \text { de las clases observadas. Los } \\
\text { textos expositivos y descriptivos fue- } \\
\text { ron utilizados por las docentes du- } \\
\text { rante sus clases en un } 38 \text { \%. } \\
\text { El docente utiliza la pregunta como } \\
\text { estrategia para generar conoci- } \\
\text { mientos } \\
\text { El } 100 \% \text { de las docentes observadas } \\
\text { empleó varias preguntas durante el } \\
\text { desarrollo de sus clases para gene- } \\
\text { rar participación y diálogo. Cabe re- } \\
\text { saltar que la mayoría de preguntas } \\
\text { formuladas por las docentes, busca- } \\
\text { ban identificar información explícita } \\
\text { de los textos, en ese orden de ideas } \\
\text { las preguntas apuntaban a desarro- } \\
\text { llar el componente semántico y sin- } \\
\text { táctico. "Durante el desarrollo de las } \\
\text { clases constantemente se realizaron } \\
\text { preguntas que generaban diversas } \\
\text { opiniones" (fragmento observación } \\
\text { de clase I. E. Sol de Oriente). }\end{array}$ & $\begin{array}{l}\text { ¿Cuál es el tipo de textos que utiliza } \\
\text { más en sus clases? } \\
\text { A pesar de que las docentes manifies- } \\
\text { tan que es importante trabajar con di- } \\
\text { ferentes tipologías textuales, el } 100 \% \\
\text { expresa una predilección por el tex- } \\
\text { to narrativo. } \\
\text { ¿Desde su planeación de lengua cas- } \\
\text { tellana, cómo articula el componente } \\
\text { pragmático de la lectura? El } 62 \% \text { de } \\
\text { las docentes entrevistadas tiene difi- } \\
\text { cultad a la hora de articular el compo- } \\
\text { nente pragmático en su planeación. El } \\
38 \% \text { lo articula por medio de diferen- } \\
\text { tes estrategias lectura, el conversato- } \\
\text { rio, la exploración de saberes previos, } \\
\text { y juegos de palabras. }\end{array}$ \\
\hline
\end{tabular}




\section{Talleres pedagógicos}

Resultados de las evaluaciones correspondientes al cierre de los talleres:

- ¿Los talleres permitieron ampliar mis conocimientos acerca del componente pragmático? $\mathrm{El} 85$ \% de las docentes calificó como excelente la ampliación de los conocimientos acerca del componente pragmático a través de los talleres y un $15 \%$ calificó este ítem como bueno.

- ¿Los ejemplos dados me sirven para generar en el aula otras estrategias de lectura que fortalezcan el componente pragmático? El 78 \% de las docentes calificó de excelente los ejemplos dados, pues les permitieron generar en el aula otras estrategias de lectura que fortalezcan el componente pragmático. El $22 \%$ lo calificó de bueno.

- ¿Cómo puedo aplicar el componente pragmático desde las diferentes áreas? Las docentes manifiestan que el componente pragmático se debe transversalizar en todas las áreas del conocimiento, por medio del diálogo, la selección de textos acordes y la formulación de preguntas que lleven al desarrollo del componente pragmático.

- Describa una estrategia que aplicaría en el aula para desarrollar el componente pragmático. Las estrategias propuestas por las docentes fueron: diseñar y resolver preguntas de tipo pragmático, relacionar los textos con el contexto de los estudiantes, utilizar la pedagogía de la pregunta, utilizar recomendaciones acerca de la pragmática aprendidas en el taller.

- Sugerencias o recomendaciones. Las docentes recomiendan continuar compartiendo el conocimiento a través de más talleres, ya que estos encuentros generan momentos de aprendizaje indispensables para el mejoramiento de las prácticas pedagógicas. También sugieren invitar a los docentes del segundo ciclo de básica primaria.

En la aplicación de los talleres se pudo notar que, aunque la investigación se desarrolló en el grado $3{ }^{\circ}$, las dificultades frente a la apropiación y desarrollo de este componente estaban presentes en todos los grados de $1 .^{\circ}$ a $3 .^{\circ}$, ya que las docentes manifestaban dificultad a la hora de comprenderlo y desarrollarlo, situación que se complicaba aún más al finalizar el ciclo. Por lo tanto, los talleres pedagógicos gozaron de mucha acogida y fueron de gran interés para la comunidad educativa.

\section{Discusión}

Se utilizó la triangulación como una técnica que nos permitió contrastar y poner en conversación cada una de las categorías con los resultados obtenidos en las técnicas de recolección de información. Para Rodríguez, Pozo y Gutiérrez: 
La triangulación implica reunir una variedad de datos y métodos referidos al mismo tema o problema. Implica también que los datos se recojan desde puntos de vista distintos y efectuando comparaciones múltiples de un fenómeno único, de un grupo, y en varios momentos, utilizando perspectivas diversas y múltiples procedimientos (2006, p. 290).

A continuación, presentamos la discusión por categoría de acuerdo al análisis de los resultados presentados.

\section{La competencia comunicativa}

El desarrollo de la competencia comunicativa es un proceso que ocurre a lo largo de la vida, pero se resaltan las primeras experiencias que nos acercan a nuestro sistema comunicativo tomando como referencia el contexto escolar, social y familiar.

Basándonos en la revisión documental de los Lineamientos curriculares del área de lengua castellana, se hace una reflexión acerca de la importancia de desarrollar la competencia comunicativa en cada individuo, ya que esta permite ponernos en relación con los demás seres, interactuar con ellos, comprender toda la información que circula a nuestro alrededor, pero ante todo, permite que el ser humano se desenvuelva con facilidad en el contexto en que se encuentre. Según Hymes:

La competencia comunicativa es el término más general para la capacidad comunicativa de una persona, capacidad que abarca tanto el conocimiento de la lengua como la habilidad para utilizarla. La adquisición de tal competencia está mediada por la experiencia social, las necesidades y motivaciones, y la acción, que es a la vez una fuente renovada de motivaciones, necesidades y experiencias (1984, p. 48).

Después de realizar las entrevistas semiestructuradas y de observar algunas prácticas de aula de las docentes del grado $3 .^{\circ}$, pudimos recoger elementos valiosos para nuestra investigación. Identificamos que las docentes utilizan algunas estrategias para desarrollar la competencia comunicativa en sus clases, tales como el diálogo constante, la pedagogía de la pregunta y ejercicios de lectura y escritura. Sin embargo, hallamos que hace falta llevar al aula estrategias que permitan a los estudiantes comprender la relación texto-contexto, y propiciar situaciones que permitan que el estudiante escriba y comparta sus ideas, realice debates y argumente sobre diferentes temas en los cuales él se ve enfrentado en su cotidianidad.

\section{Componente pragmático}

La competencia comunicativa se refiere al conocimiento de la lengua y su habilidad para ponerla en contexto y el componente pragmático hace parte de esta competencia, el cual según María Victoria Escandell (1996, p. 22) es el 
estudio de los principios que regulan el uso del lenguaje en la comunicación y está relacionado con la intencionalidad de lo que se dice, por qué, a quién y para que se dice.

$\mathrm{Al}$ analizar las observaciones y la entrevista semiestructurada en las que se indaga acerca de los criterios metodológicos y el conocimiento que tiene el docente sobre el componente pragmático, hallamos que las docentes en sus clases enfocan sus estrategias al desarrollo del componente semántico y sintáctico, y realizan preguntas literales sin abordar el propósito comunicativo del texto. "En la mayoría de las clases se enfocaron al desarrollo del componente semántico y sintáctico. En una de las clases se observa unas estrategias enfocadas al desarrollo del componente pragmático" (fragmento observación de clase I. E. José Acevedo y Gómez).

Igualmente, dentro de su conocimiento disciplinar se hace necesario seguir ampliando sus saberes acerca del componente pragmático, teniendo en cuenta las orientaciones ministeriales de lengua castellana "el componente pragmático es el conocimiento de un tema como la lectura" (fragmento observación de clase I. E. Luis Carlos Galán Sarmiento). Según el MEN, en el documento Matriz de referencia, grado tercero área de lenguaje, en el proceso de lectura en el componente pragmático los estudiantes deben evidenciar aprendizajes tales como: "evaluar y reconocer elementos e información explícita o implícita de la situación comunicativa, caracterizar el enunciador respecto a lo enunciado, identificar la función social de algunos textos de circulación cotidiana e identificar intenciones, propósitos y perspectivas" (MEN, 2006, p. 3).

Estas referencias alimentan la experiencia didáctica del docente, pero deben ser ampliadas con otras fuentes de conocimiento y de saberes como Escandell Vidal (1996) y Reyes (1994) quienes son referentes importantes de la pragmática en la actualidad, pues el profesor debe estar en una constante actualización y estar a la vanguardia en términos del saber que enseña y de didáctica en general.

\section{Función social de la lectura}

En los procesos de lectura, juega un papel significativo el contexto, por tanto, "en la enseñanza del área de lenguaje, además de enfatizar en los aspectos estructurales, se debe hacer énfasis en sus dimensiones sociales" (MEN, 1998). Una vez realizadas las observaciones de clase a las docentes participantes en la investigación, desde la categoría función social se evidenció que la totalidad de las docentes utilizó un léxico claro y acorde al nivel de los estudiantes. Sin embargo, esta estrategia permitió que los estudiantes identificaran aspectos literales del texto, ya que sus respuestas siempre estuvieron ligadas a tales aspectos. Según Kalmam: 
La preocupación fundamental que subyace al estudio de intervención en eventos de lectura y escritura se aborda desde un interés por comprenderlas como prácticas sociales más que como un conjunto de destrezas que se centran en la manipulación mecánica de elementos aislados del texto (2003, p. 43).

Por tanto, se hace indispensable que además de usar un léxico familiar a los estudiantes, en el desarrollo de las clases se profundice también en otras estrategias didácticas que permitan relacionar los conocimientos con la función social que tienen, para que así el estudiante le encuentre sentido a lo que lee. $\mathrm{Al}$ analizar las entrevistas y las observaciones de clase, las docentes manifestaron la importancia de integrar en sus prácticas pedagógicas la lectura con el contexto. En algunas de sus clases pudimos observar que aplicaban estrategias como la exploración de saberes previos, diálogo sobre sus experiencias relacionadas con el texto, proposición de soluciones a problemas de su entorno y la elección de lecturas que partieran de los intereses de los estudiantes.

Por lo anterior se puede afirmar que, por parte de la mayoría de las docentes, existe un conocimiento claro de la importancia que tiene en los procesos de enseñanza aprendizaje establecer una relación entre los textos y el contexto de los estudiantes en función de la situación comunicativa. Sin embargo, en la práctica se debe continuar fortaleciendo procesos a través de la cualificación docente que permitan hacer mayor énfasis en las dimensiones sociales de la lectura y no solo en las estructurales de la lengua. Como afirma Kalman, la "lectura debe ser comprendida como una práctica social más que como un conjunto de destrezas que se centran en la manipulación mecánica de elementos aislados del texto" (2003, p. 38).

\section{Conocimiento disciplinar y didáctico}

Con esta investigación se verificaron algunos planteamientos de Perafán (2013) quien define el conocimiento profesional del docente como un sistema de ideas integradas. Los resultados arrojados en las observaciones nos demuestran que las docentes investigadas priorizan los saberes basados en la experiencia y sus rutinas, las cuales se ven evidenciadas en la forma como dictan sus clases y en la manera como utilizan reiterativamente alguna tipología textual ya que en las cuatro observaciones se utilizó el texto narrativo.

$\mathrm{Al}$ analizar los resultados de las entrevistas diseñamos talleres que, al aplicarlos, permitieron reconocer las fortalezas y dificultades que presentaban las docentes al identificar preguntas de tipo pragmático. Por esta razón, se enfatizó en las cuatro etapas epistemológicas propuestas por Perafán: "los saberes académicos, las teorías implícitas, los saberes basados en la experiencia y los guiones y rutinas" (Perafán, 2013, p. 2). Teniendo en cuenta que el saber acadé- 
mico y las teorías implícitas son indispensables para cualificar la labor docente, se hizo necesario fortalecer la conceptualización del componente pragmático.

Los saberes basados en la experiencia están fundamentados en la práctica profesional del docente; por ello, se realizaron ejercicios de ejemplificación, comparación y elaboración de preguntas de carácter pragmático en donde las participantes de una manera experimental se pudieron acercar a la estructura de este tipo de preguntas. De esta manera, cuando se les preguntó: ¿cómo puedo desarrollar en mis estudiantes el componente pragmático?, las docentes dieron a conocer diferentes estrategias para poner en práctica el componente pragmático, como relacionar las lecturas con el contexto en el que se desenvuelven los estudiantes y utilizar diferentes tipos de texto e identificar las marcas que los acercan a dicho componente.

Los guiones y rutinas hacen parte del conocimiento y cotidianidad del profesor; por ello, con la construcción de las actividades referidas al componente pragmático, las docentes pudieron ampliar su perspectiva acerca de la labor en el aula y vieron la necesidad de actualizar y mejorar día a día su práctica respecto a la competencia comunicativa. Incluso recomiendan continuar compartiendo el conocimiento a través de más talleres, ya que estos encuentros generan momentos de aprendizaje indispensables para el mejoramiento de las prácticas pedagógicas.

A través de la metodología utilizada en la investigación se pudo conocer, interpretar y comprender el medio donde se encuentran inmersas las instituciones participantes, se realizó observación de las prácticas pedagógicas, permitiendo explorar y transformar algunas concepciones sobre el componente pragmático. Las ventajas de las técnicas de recolección de información fueron las siguientes: con la revisión documental se recolectaron datos referentes a la competencia comunicativa y al componente pragmático, que permitió enriquecer el conocimiento didáctico de las ejecutoras y participantes de la investigación. Con la observación se identificaron las estrategias utilizadas por los docentes para el desarrollo del componente pragmático, con la entrevista se identificaron los conocimientos de los docentes respecto a este componente, y con los talleres se orientaron las prácticas de lectura sobre el tema de investigación.

La desventaja en nuestro proceso investigativo estuvo en la aplicación de los talleres pedagógicos, ya que no se encontraron estrategias metodológicas claras para el desarrollo del componente pragmático. Por lo anterior se hizo necesario retomar elementos de las guías de aplicación, de las cartillas de pruebas Saber y de nuestra propia autoría para formular e identificar preguntas de tipo pragmático que sirvieran de guía a las docentes. Estas fueron: ¿quién lo dijo?, ¿a quién se dice?, ¿quién narra? (permiten reconocer las voces en el texto), ¿por 
qué lo hizo?, ¿por qué lo dijo? (sirve para caracterizar personajes), ¿para qué es el texto?, ¿cuál es la intención de quien enuncia?, ¿qué papel social cumple este tipo de texto?, ¿para qué se dice?, ¿qué hace el texto? (permiten identificar el propósito comunicativo), según el texto podemos afirmar que: (se identifica la información explícita) y ¿qué elegirías?, ¿para qué utilizarías el texto? (permite seleccionar información pertinente del texto).

\section{Conclusiones}

A pesar de la existencia de las orientaciones curriculares y pedagógicas del Ministerio de Educación Nacional en el área de lengua castellana, falta tenerlas en cuenta en el desarrollo de las clases. Esto sucede debido a que la labor profesional del docente está sujeta a los guiones y rutinas, los cuales se vuelven esquemáticos y dificultan la posibilidad de ampliar las perspectivas didácticas. Lo anterior se corrobora en la aplicación de las técnicas de recolección de información.

La apropiación que hace el docente de las orientaciones ministeriales para el área de lenguaje no genera interacción con el saber científico que es la competencia pragmática ya que, según los resultados, no hay suficiente apropiación y reconocimiento de los tres componentes de la competencia comunicativa, porque el hecho de ser docente no significa que se esté preparado científicamente en todas las disciplinas que enseña.

En las cuatro instituciones educativas participantes en el proceso investigativo, los aprendizajes por mejorar en las pruebas Saber durante este periodo giraban en torno al componente pragmático y, aunque por tres años seguidos el Icfes ha dado la misma recomendación de mejorar en el reconocimiento de la información implícita y explícita dentro de la situación comunicativa, los avances siguen siendo mínimos. Estos informes revelan una fortaleza mayor en las prácticas de enseñanza desde los componentes semántico y sintáctico en relación con el pragmático (ver tabla 1, aprendizajes por mejorar).

En cuanto a las estrategias que utilizan las docentes del grado $3 .^{\circ}$ para la enseñanza de la lectura en el componente pragmático se pudo concluir que deben ser enriquecidas y usadas con mayor frecuencia, ya que las docentes en sus prácticas pedagógicas priorizan en la identificación de estructuras textuales (componente sintáctico) y el sentido o significación del texto (componente semántico), dejando de lado la función social y los propósitos comunicativos del texto (componente pragmático). Igualmente, la mayoría de las actividades de aula están encaminadas en dar razones literales y explícitas de la información abordada en los textos, más que en llegar a descubrir elementos implícitos y la función social del tipo de texto que se aborda. 
Las docentes le dan prioridad al texto narrativo, limitando el uso de otras tipologías textuales, dejando de lado las orientaciones de los Estándares básicos de competencia del área de lenguaje, los cuales expresan que los estudiantes deben leer diferentes clases de textos, reconociendo su función social, identificando el propósito comunicativo y la idea global de un texto.

También se puede concluir que los docentes reconocen la importancia del componente pragmático en la lectura, pero aún se requiere profundizar en elementos metodológicos y conceptuales que les permitan aplicarlos. Para ello debe seguir enriqueciendo su conocimiento disciplinar y su práctica de aula.

En general, se pudo evidenciar que las prácticas de enseñanza de la lectura desde el componente pragmático en el grado $3 .^{\circ}$ de las cuatro instituciones analizadas se desarrollan a través de estrategias como el diálogo constante, la pedagogía de la pregunta y ejercicios de lectura y escritura, las cuales deben ser fortalecidas para permitir una mayor apropiación de la función comunicativa que tiene un texto.

\section{Agradecimientos}

Este artículo presenta los resultados del trabajo de investigación denominado El componente pragmático: elemento indispensable para la lectura en contexto, realizado durante la Maestría en Educación, en la línea didáctica de la lectura y la escritura de la Universidad de Medellín. La investigación fue orientada por la profesora Luz Adriana Restrepo Calderón, magíster en Docencia Lenguaje y Educación de la Universidad de Antioquia, y bajo la asesoría de la profesora Laura Marcela Quintero Montoya, magíster en Formación de Profesores de Español como Lengua Extranjera.

\section{Referencias}

Cifuentes, R. (2011). Diseño de proyectos de investigación cualitativa. Buenos Aires: Noveduc

Colomer, T. (2001). La enseñanza de la literatura como construcción de sentido. Lectura y Vida, Revista Latinoamericana de Lectura, 19.

Escandell, M. (1996). Introducción a la pragmática. Barcelona: Ariel.

Hymes, D. (1984). Hacia etnografías de la educación. En P. Garvin y Y. Lastra. (Eds.), Antología de estudios de etnolingüística y sociolingüística (pp. 48-87). Ciudad de México: Programa Editorial de la Coordinación de Humanidades. Universidad Autónoma de Mexico.

Kalman, J. (2003). El acceso a la cultura escrita: la participación social y la apropiación de conocimientos en eventos cotidianos de lectura y escritura. Revista Mexicana de Investigación Educativa, 8(17), 37-66. 
Ministerio de Educación Nacional. (2006). Estándares básicos de competencias. Bogotá: Ministerio de Educación Nacional.

Ministerio de Educación Nacional. (2016). Guía lineamientos para las aplicaciones muestral y censal. Bogotá: Ministerio de Educación Nacional.

Ministerio de Educación Nacional (1998). Lineamientos curriculares. Bogotá: Ministerio de Educación Nacional.

Perafán, G. (2013). La transposición didáctica como estatuto epistemológico fundante de los saberes académicos del profesor. Folios, (37), 83-93. doi: https://doi.org/10.17227/01234870.37folios83.93

Perafán, G. (2014). Aspectos generales y primeros avances para el encuadre de la investigación sobre el conocimiento profesional específico del profesorado. Educación, 23(44) 48-64. Recuperado de http://revistas.pucp.edu.pe/index.php/educacion/article/view/8940

Reyes, G. (1994). La pragmática lingüística: el estudio del uso del lenguaje . Barcelona: Montesinos.

Rodríguez, C., Pozo, T. y Gutiérrez, J. (2006). La triangulación analítica como recurso para la validación de estudios de encuesta recurrentes e investigaciones de réplica en educación superior. Relieve, 12(2), 289-305. Recuperado de http://www.uv.es/RELIEVE/v12n2/RELIEVEv12n26.htm 